\title{
A new chemotaxis assay shows the extreme sensitivity of axons to molecular gradients
}

William J Rosoff, Jeffrey S Urbach, Mark A Esrick, Ryan G McAllister, Linda J Richards \& Geoffrey J Goodhill Nature Neuroscience 7, 678-682 (2004)

The production process introduced several errors into Fig. $3 \mathrm{c}$. A minus sign was omitted from the negative value of " 0.05 " on the $y$-axis, and the

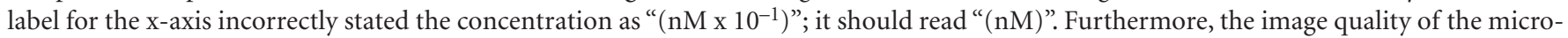

graphs in Fig. 3d-g was poor due to color conversion problems. The corrected Fig. 3 is shown below.
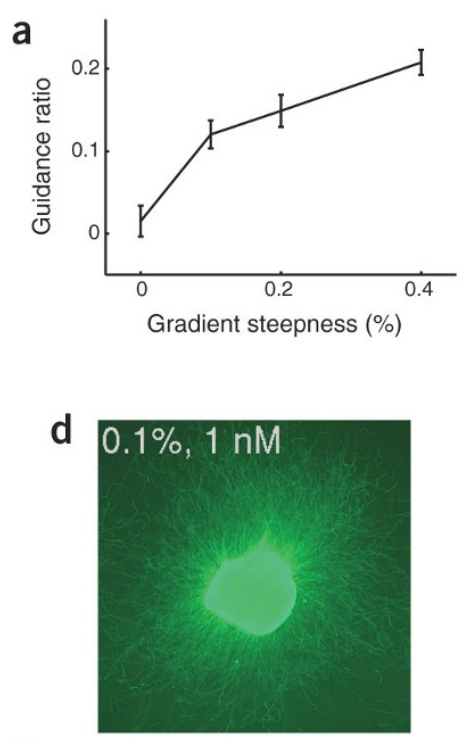

g

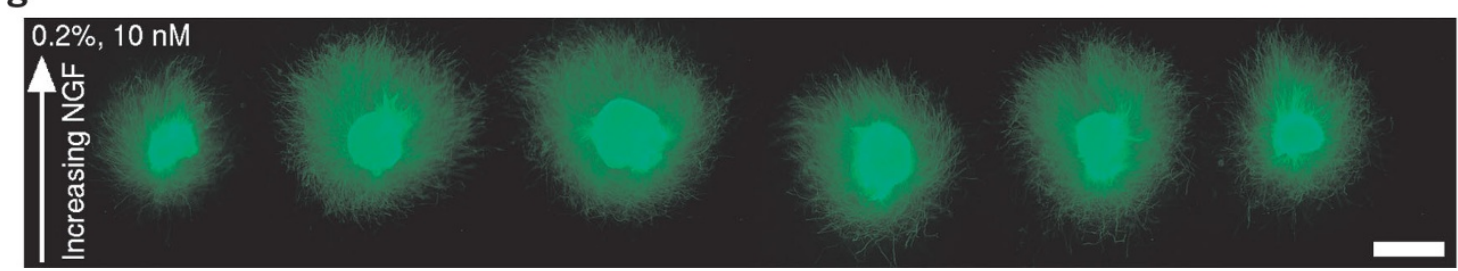

Figure 3 Guidance of DRG axons by NGF gradients. (a) Guidance ratio of DRG explants as a function of gradient steepness. Error bars are s.e.m. For $s=0.1 \%$, the guidance ratios were significantly different from zero $\left(P<10^{-8}\right)$, and $80 \%$ of explants $(43$ of 54$)$ had positive guidance ratios. Responses for $s=0.2 \%$ and $0.4 \%$ were also significantly different $(P<0.005)$. For $s=0.4 \%, 53$ of 54 explants had positive guidance ratios ( 54 explants per condition pooled over three separate experiments; similar results were seen in each experiment). (b) Outgrowth in response to NGF after 36-40 h in culture, as measured by the number of bright pixels representing neurites, divided by the area of the explant (36 explants per condition pooled over three separate experiments). (c) Guidance as a function of absolute NGF concentration at the explants for $s=0.2 \%$. The probability that the mean guidance ratio is zero in each case is given above each data point (54-108 explants per condition pooled over six separate experiments). Although the $1 \mathrm{nM}$ condition represents equivalent conditions to the $0.2 \%$ condition in a, the guidance ratios in these two cases are not exactly the same as they are derived from a different series of experiments. (d-f) Typical explants from experiment in a for $s=0.1 \%, 0.2 \%$ and $0.4 \%$, respectively (guidance ratios within 0.01 , that is $5-10 \%$, of the mean). The NGF gradient points upwards. (g) Complete row of explants for one dish from experiment in $\mathbf{c}, s=0.2 \%, 10 \mathrm{nM}$. Clear turning of the neurites is apparent. Scale bars, $500 \mu \mathrm{m}$. 